Vol. 26, Issue 4, August 2003

Editorial

323 Geriatric Oncology: Appropriate Assessment is the Basis for Clinical Trials and Routine Care Bokemeyer, C. (Tübingen); Kolb, G. (Lingen)

Original Articles

325 Concomitant Gemcitabine (Gemzar ${ }^{\circledR}$ ) and Extended Nodes Irradiation in the Treatment of Pancreatic and Biliary Carcinoma: A Phase I Study

Morganti, A.G.; Trodella, L.; Valentini, V.; Macchia, G.; Alfieri, S.; Smaniotto, D.; Luzi, S.; Costamagna, G.; Doglietto, G.B.; Cellini, N. (Rome)

330 Recurrence after Radical Prostatectomy for OrganConfined Prostate Cancer

Scheepe, J.R.; Vis, A.N. (Rotterdam); Mickisch, G.H. (Bremen)

334 Manifestation, Latency and Management of Late Urological Complications After Curative Radiotherapy for Cervical Carcinoma

Gellrich, J.; Hakenberg, O.W.; Oehlschläger, S.; Wirth, M.P. (Dresden)

Casuistic Contributions

341 Solitary Bone Metastasis from Myxoid Liposarcoma Mai, S.K.; Wenz, F.; Voggenreiter, G.; Neff, K.W.; Diehl, S.; Düber, C. (Mannheim)

344 Acute Pseudo-Obstruction of the Small Intestine Following High-Dose Chemotherapy and Stem-Cell Support

Bauernhofer, T.; Ploner, F.; Kuss, I.; Samonigg, H.; Eibl, M.; Sill, H.; Pfeifer, J. (Graz)

348 Acute Pericarditis and Pleural Effusion Complicating Cytarabine Chemotherapy

Gähler, A.; Hitz, F.; Hess, U.; Cerny, T. (St. Gallen)

Review Articles

351 Treatment of CNS Lymphoma with the Anti-CD20 Antibody Rituximab: Experience with Two Cases and Review of the Literature Pels, H. (Bonn); Schulz, H. (Köln); Schlegel, U. (Bonn); Engert, A. (Köln)
Band 26, Heft 4, August 2003

Editorial

323 Geriatrische Onkologie: Angemessenes Assessment ist die Basis für Studien und den klinischen Alltag Bokemeyer, C. (Tübingen); Kolb, G. (Lingen)

Originalarbeiten

325 Kombinierte Behandlung von Pankreas- und Gallengangskarzinomen mit Gemcitabin (Gemzar®) und Lymphknotenbestrahlung: Eine Phase-I-Studie Morganti, A.G.; Trodella, L.; Valentini, V.; Macchia, G.; Alfieri, S.; Smaniotto, D.; Luzi, S.; Costamagna, G.; Doglietto, G.B.; Cellini, N. (Rome)

330 Progression nach radikaler Prostatektomie wegen organbegrenztem Prostatakarzinom Scheepe, J.R.; Vis, A.N. (Rotterdam); Mickisch, G.H. (Bremen)

334 Manifestation, Latenzzeit und Behandlung urologischer Spätkomplikationen nach kurativer Strahlentherapie des Zervixkarzinoms

Gellrich, J.; Hakenberg, O.W.; Oehlschläger, S.; Wirth, M.P. (Dresden)

Kasuistiken

341 Solitäre Knochenmetastase bei myxoidem Liposarkom Mai, S.K.; Wenz, F.; Voggenreiter, G.; Neff, K.W.; Diehl, S.; Düber, C. (Mannheim)

344 Akute Pseudo-Obstruktion des Dünndarms nach Hochdosis-Chemotherapie und Stammzelltransplantation Bauernhofer, T.; Ploner, F.; Kuss, I.; Samonigg, H.; Eibl, M.; Sill, H.; Pfeifer, J. (Graz)

348 Cytosar-assoziierter akuter Perikard- und Pleuraerguss

Gähler, A.; Hitz, F.; Hess, U.; Cerny, T. (St. Gallen)

Übersichtsarbeiten

351 Behandlung primär zerebraler Lymphome mit dem anti-CD20-Antikörper Rituximab: Erfahrungen mit zwei eigenen Fällen und Literaturübersicht Pels, H. (Bonn); Schulz, H. (Köln); Schlegel, U. (Bonn); Engert, A. (Köln)

\begin{tabular}{|c|c|}
\hline KARGER & (C) 2003 S. Karger GmbH, Freiburg \\
\hline $\begin{array}{l}\text { Fax }+497614520714 \\
\text { E-mail Information@Karger.de } \\
\text { www.karger.com }\end{array}$ & $\begin{array}{l}\text { Artikel (Volltext) und Inhaltsverzeichnisse } \\
\text { sowie das vorläufige Inhaltsverzeichnis des nächsten Heftes: } \\
\text { www.karger.com/onk_bk.htm }\end{array}$ \\
\hline
\end{tabular}




\section{Vol. 26, Issue 4, August 2003}

355 Comprehensive Geriatric Assessment in the Elderly Cancer Patient

Friedrich, C.; Pientka, L. (Bochum); Kolb, G. (Lingen/Ems); Wedding, U. (Jena)

\section{Perioperative Chemotherapy in Advanced Bladder Cancer: Part I Neoadjuvant Treatment Goebell, P.J.; Otto, T.; Rübben, H. (Essen)}

366 Therapy of Cutaneous Lymphoma - Current Practice and Future Developments

Dummer, R.; Kempf, W.; Hess Schmid, M.; Häffner, A.; Burg, G. (Zürich)

373 Treatment of Relapsed Acute Promyelocytic Leukemia Lengfelder, E.; Gnad, U. (Mannheim); Büchner, T. (Münster); Hehlmann, R. (Mannheim)

Reports of Oncological Societies

380 Deutsche Krebsgesellschaft e.V.

Abstracts

381 Oral Presentations at the Symposium 'Novel Approaches for the Discovery of Anticancer Agents', Freiburg,

Germany, June 18-21, 2003

\author{
400 Book Reviews \\ 404 PharmaForum \\ 408 Industrial Forum \\ 412 Meetings an Conferences \\ 318 Imprint \\ 415 Guidelines for Authors
}

Prospects for contents of the next issues are given on page 416.

\section{Band 26, Heft 4, August 2003}

355 Geriatrisches Assessment beim älteren onkologischen Patienten

Friedrich, C.; Pientka, L. (Bochum); Kolb, G. (Lingen/Ems); Wedding, U. (Jena)

361 Perioperative systemische Therapie des fortgeschrittenen Harnblasenkarzinoms: Teil I neoadjuvante Therapie Goebell, P.J.; Otto, T.; Rübben, H. (Essen)

366 Therapie kutaner Lymphome - aktuelle Verfahren und zukünftige Entwicklungen

Dummer, R.; Kempf, W.; Hess Schmid, M.; Häffner, A.; Burg, G. (Zürich)

373 Behandlungsmöglichkeiten bei rezidivierter akuter Promyelozytenleukämie

Lengfelder, E.; Gnad, U. (Mannheim); Büchner, T. (Münster); Hehlmann, R. (Mannheim)

Mitteilungen onkologischer Gesellschaften

380 Deutsche Krebsgesellschaft e.V.

Abstracts

381 Vorträge beim Symposium «Novel Approaches for the Discovery of Anticancer Agents», Freiburg i.Br., 18.-21. Juni 2003

400 Buchbesprechungen

404 PharmaForum

408 Industrieforum

412 Tagungen und Kongresse

318 Impressum

U3 Hinweise für Autoren (3. Umschlagseite)
Einen Ausblick auf den Inhalt der kommenden Hefte finden Sie auf Seite 416.

\section{KARGER}

\section{(C) 2003 S. Karger GmbH, Freiburg}

Artikel (Volltext) und Inhaltsverzeichnisse

E-mail Information@Karger.de

www.karger.com

sowie das vorläufige Inhaltsverzeichnis des nächsten Heftes: www.karger.com/onk_bk.htm 